\title{
Reconhecimento de atividades humanas baseado na análise de fluxo contínuo de dados simbólicos
}

\author{
Wesllen Sousa Lima \\ Eduardo J. P. Souto \\ wesllen@icomp.ufam.edu.br \\ esouto@icomp.ufam.edu.br \\ Instituto de Computação na Universidade Federal do Amazonas \\ Manaus, Amazonas
}

\begin{abstract}
Smartphones sensing capabilities have enabled the development of Human Activity Recognition (HAR) solutions for better understanding human behavior through computational techniques. However, these solutions have been difficult to perform in dynamic scenarios because they do not observe data evolution over time and the high consumption of computational resources, such as memory, processing and energy. This occurs because the HAR problem for smartphones has been solved through classification models generated by offline machine learning algorithms that, in this case, are limited by a data history with little information about human activities. The problem with this approach is that human activities change constantly over time and are strongly influenced by the physical environment and the user's profile. To overcome these problems this doctoral thesis proposes a new approach to recognize human activities based on the symbolic data streaming analysis. Our approach enables the development of low-cost, scalable HAR systems capable of adapting to data change over time. In this context, this thesis proposes a framework called DISTAR (DIscrete STream learning for Activity Recognition), responsible for standardizing the analysis of data stream process and generation of adaptive models that observe the data evolution over time without storing a data history. The DISTAR framework uses the symbolic representation algorithms known for reducing the dimensionality and numerosity of the data. In addition, this thesis also proposes a new adaptive online algorithm, called NOHAR (NOvelty discrete data stream for Human Activity Recognition), which uses as basis the DISTAR framework. Experimental results using three databases show that NOHAR is 13 times faster compared to the state of the art and is able to reduce memory consumption by an average of 99.97
\end{abstract}

\section{KEYWORDS}

Human Activity Recognition, Data Streaming, Symbolic Representation Algorithm, Inertial Sensors

In: II Concurso de Teses e Dissertações (CTD 2020), São Luís, Brasil. Anais Estendido do Simpósio Brasileiro de Sistemas Multimídia e Web (WebMedia). Porto Alegre Sociedade Brasileira de Computação, 2020.

(c) 2020 SBC - Sociedade Brasileira de Computação.

ISSN 2596-1683

\section{INTRODUÇÃO}

Este artigo apresenta um resumo dos resultados obtidos na tese defendida no Programa de Pós-graduação em informática no Instituto de Computação da Universidade Federal do Amazonas em 05/04/2019. O trabalho foi desenvolvido pelo 10 autor, num período de 48 meses, sob a orientação do último autor.

A área de Reconhecimento de Atividade Humana (HAR) tem sido abordada na literatura como um problema típico de reconhecimento de padrões, que tenta identificar a atividade que está sendo realizada por um indivíduo em um determinado momento. Esse problema foi comumente resolvido usando algoritmos de aprendizado de máquina offline tradicionais, como algoritmos rasos (por exemplo, SVM e KNN) [8, 12, 27] e algoritmos de aprendizado profundo (por exemplo, DNN e CNN) [14, 19, 30]. No contexto do HAR, os modelos offline são computacionalmente caros, além de serem incapazes de se adaptar às mudanças de dados continuamente recebidos. Os dados usados para reconhecer as atividades humanas mudam constantemente ao longo do tempo devido às mudanças no comportamento humano como estado de saúde dos indivíduos e ambientes físicos.

Quando o reconhecimento da atividade é realizado via smartphones, o problema é ainda pior, pois os dados de sensores devem ser constantemente coletados e analisados em tempo real e os recursos computacionais dos smartphones são limitados por memória e processamento. Em geral, as soluções HAR baseadas em smartphones tendem a contar com serviços externos para armazenar histórico de dados e gerar modelos de classificação [3, 6, 17]. No entanto, essa prática também tem desvantagens para smartphones, pois a interface de rede sem fio consome muita energia da bateria. Esses problemas dificultam a aplicação de algoritmos offline em sistemas HAR baseados em smartphones.

Os problemas destacados acima levaram a comunidade de pesquisa de HAR a se mover em uma nova direção com base em sistemas de aprendizagem online [1, 2, 11, 31]. Esses sistemas empregam algoritmos online projetados para analisar o fluxo de dados em diferentes velocidades, escalas e granularidades [10]. A estratégia usada permite que os modelos HAR online sejam atualizados dinamicamente quando novos dados são coletados. Os algoritmos online não dependem do histórico de dados para gerar modelos porque apenas informações de dados fundamentais, como medidas estatísticas, são extraídas e armazenadas na memória, tornando-os capazes de operar com uma pequena quantidade de memória. Assim, sempre que os dados brutos contidos na memória são processados, a memória é liberada para armazenar os novos dados coletados do fluxo contínuo de dados [7]. 
Paralelamente ao uso de algoritmos online, o Sousa Lima et al. [28] propôs modelos de classificação offline de baixo custo baseados em algoritmos de representação simbólica. Esse tipo de algoritmo compacta os dados brutos em um conjunto de símbolos representados por um pacote de palavras. Cada palavra é representada por sua frequência (histograma) na série temporal. Então, encontrar padrões nos dados discretizados envolve medir (des) similaridade entre histogramas. Na prática, ocorre uma redução na dimensionalidade dos dados, onde as palavras representam uma faixa fixa de valores contínuos. A literatura apresenta dois algoritmos de representação simbólica que podem ser utilizados para discretizar os dados, o Symbolic Aggregate Approximation (SAX) [16] e o Symbolic Fourier Approximation (SFA) [23]. Sousa Lima et al. [28] mostrou que esses dois algoritmos podem reduzir o consumo de memória em até $94,48 \%$. Além disso, os algoritmos de classificação offline adaptados para lidar com dados simbólicos (por exemplo, SAX-VSM [24] e BOSS [22] são em média 6 vezes mais rápidos do que os algoritmos offline tradicionais no contexto HAR.

A combinação entre aprendizagem online e algoritmos de representação simbólica pode ajudar a resolver dois problemas da área de HAR. O primeiro é sobre modelos dinâmicos que lidam com dados de entrada contínuos. O segundo trata de algoritmos de baixo custo. A fusão dessas duas técnicas dá origem a uma categoria sem precedentes de algoritmos de aprendizagem chamados algoritmos simbólicos online. Para ajudar a entender como um algoritmo simbólico online funciona, propusemos o primeiro framework conceitual, denominado DISTAR (DIscrete STream learning for Activity Recognition), para auxiliar futuras propostas de construção de um algoritmo simbólico online. A ideia do DISTAR é mostrar como os fluxos de dados podem ser segmentados e transformados em símbolos usando algoritmos de discretização.

Para validar as ideias propostas no DISTAR, apresentamos um novo algoritmo simbólico online denominado NOHAR (NOvelty discrete data stream for Human Activity Recognition). NOHAR é um algoritmo detector de novidades capaz de detectar novas atividades monitorando a mudança de conceitos em histogramas de palavras. Especificamente, NOHAR classifica histogramas de padrão em três tipos: histogramas desconhecidos (uBOP), histogramas conhecidos/referência (rBOP) e novos histogramas (nBOP). Como baseline, comparamos os resultados apenas com o algoritmo AnyNovel [2] porque é o único algoritmo online detector de novidades criado para HAR. Os experimentos foram realizados nos conjuntos de dados UCI-HAR [5], WISDM [13] e SHOAIB [26]. Os resultados mostram que o NOHAR é 33 vezes mais rápido e quase $100 \%$ mais eficiente em termos de memória comparado ao algoritmo AnyNovel [2].

Este artigo está organizado da seguinte forma: A seção 2 apresenta os conceitos do framework DISTAR. A seção 3 apresenta detalhes das etapas do algoritmo NOHAR. A seção 4 descreve o protocolo experimental usado para avaliar os algoritmos NOHAR e AnyNovel e a seção 5 apresenta os resultados. Finalmente, a seção 6 apresenta as principais conclusões da pesquisa.

\section{FRAMEWORK DISTAR}

Antes de descrever a estrutura do DISTAR, apresentaremos brevemente alguns conceitos. Em geral, os algoritmos online atuam em uma quantidade limitada de memória, aqui chamada de buffer. $\mathrm{O}$ buffer consiste em uma pequena unidade de memória volátil usada para armazenar uma quantidade limitada de dados. Um buffer pode ser dividido em unidades de memória menores chamadas chunks. Cada chunk é representado por uma janela de tempo. Os dados contidos nos chunks passam por um processo de discretização e são transformados em palavras (símbolos). Por fim, as palavras são representadas por um histograma.

No DISTAR, os histogramas são vistos como Bag-of-Pattern (BOP). Um BOP pode conter informações suficientes sobre as atividades e ações dos usuários. Nesse caso, o papel do algoritmo de aprendizagem é analisar, detectar e reconhecer padrões nos BOPs gerados a partir do fluxo contínuo de dados. O DISTAR classifica os BOPs em três categorias: i) BOP desconhecido (uBOP) - quando os histogramas são desconhecidos; ii) BOP Novo (nBOP) - quando os histogramas apresentam alguma novidade ou novo conceito nos dados; e iii) BOP de referência (rBOP) - quando o histograma é conhecido pelo modelo de classificação. A Figura 1 exemplifica as etapas de discretização de dados brutos e geração de BOPs no DISTAR.

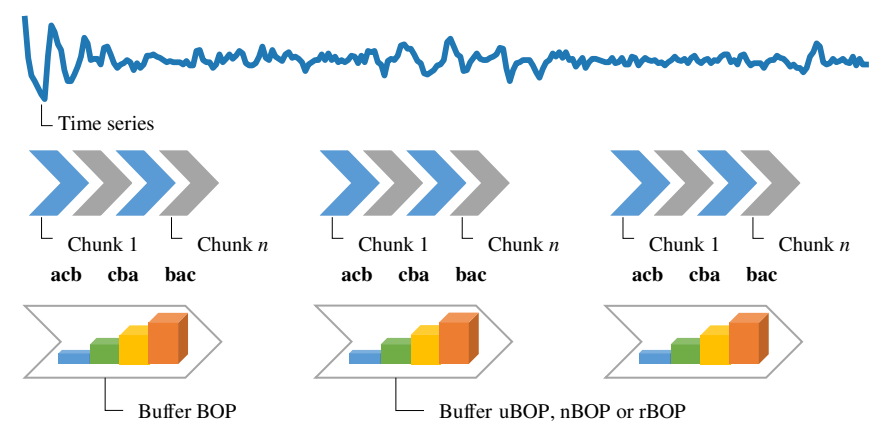

Figure 1: Visão geral das etapas de discretização de dados brutos e geração de BOPs no framework DISTAR.

A etapa de discretização visa compactar dados brutos para reduzir a dimensionalidade e otimizar o uso da memória do dispositivo. Para isso, pode ser utilizado qualquer discretizador, como os algoritmos de representação simbólica SAX [16], iSAX [25] ou SFA [23]. A segunda etapa é organizar os dados discretizados em BOPs. Cada BOP pode representar um padrão que deve ser abordado na etapa de classificação e aprendizagem. A etapa de classificação discrimina os histogramas uBOP, nBOP e rBOP.

A fase de aprendizagem consiste na análise dos histogramas uBOP, nBOP ou rBOP. Nesta etapa, analisamos se há mudanças na distribuição de frequência dos histogramas causadas, por exemplo, por alguma mudança no comportamento do usuário. Esta mudança é conhecida no campo da aprendizagem online como Concept Drift [2]. Quando o algoritmo detecta uma mudança na distribuição de dados, o modelo de classificação é atualizado. Na prática, o algoritmo compara se as distribuições de frequência entre dois histogramas são semelhantes, observando dois limites (limites de confiança e tolerância). A análise desses limites pode levar a três caminhos: (i) se a taxa de similaridade estiver dentro de um limite de confiança, então não há alteração nos dados; ii) se a taxa de similaridade estiver fora do limite de confiança, então os histogramas são diferentes; iii) por último, se a taxa de similaridade estiver entre o limite de confiança e 
o limite de tolerância, então os dados podem estar evoluindo. O limite de tolerância identifica mudanças de conceito nos histogramas uBOP, nBOP e rBOP. Portanto, o histograma deve receber ajustes de frequência nas palavras para se adaptar às mudanças ao longo do tempo.

A fase de aprendizagem também é responsável por controlar o ciclo de vida dos histogramas. Por exemplo, um uBOP pode se tornar um nBOP se esse histograma reaparecer com o tempo e a frequência de repetição exceder um limite definido como parâmetro. Para o nBOP, a diferença é que o usuário deve rotular o histograma por meio do aprendizado ativo. Quando a frequência de repetição de um nBOP atinge um limite, esse histograma é transformado em um rBOP e o modelo de classificação é atualizado. Além disso, DISTAR pode detectar e esquecer atividades obsoletas, ou seja, atividades que não são mais realizadas pelo usuário. Na prática, os histogramas que representam essas atividades são excluídos automaticamente do modelo.

\section{ALGORITMO NOHAR}

O algoritmo NOHAR é baseado no framework DISTAR. O NOHAR está focado na análise de séries temporais extraídas de sensores inerciais. Todos os sensores inerciais retornam matrizes multidimensionais com valores distribuídos em três eixos de coordenadas (x, y e z).

A fase de discretização ocorre em quatro etapas. Primeiro, os dados são segmentados em chunks. Em seguida, um filtro de suavização Exponencial e Lowpass [20] deve ser aplicado com o objetivo de remover ruídos nos dados. Em seguida, os chunks são submetidos ao processo de discretização usando o algoritmo SAX. Por fim, informações estatísticas são extraídas dos chunks (média e variância) e vinculadas às palavras correspondentes a cada chunk. Essa estratégia visa fornecer características adicionais ao modelo de classificação na tentativa de melhorar a discriminação das atividades humanas.

A fase de geração do histograma é composta por três etapas. A primeira etapa consiste no alinhamento das palavras. Nesta etapa, as palavras que representam os mesmos segmentos são mapeadas em um dicionário. Na segunda etapa, novas palavras são criadas e adicionadas a um histograma existente ou, para as palavras existentes, as frequências são atualizadas no histograma. Por fim, as palavras contidas nos histogramas são ordendas em ordem crescente para facilitar a comparação de histogramas na fase de aprendizagem. A figura 2 mostra um exemplo de histograma gerado por NOHAR.

A etapa de aprendizagem do algoritmo NOHAR corresponde ao componente de adaptação do framework DISTAR. Esta fase consiste na busca de histogramas semelhantes ao novo histograma gerado após o processamento contínuo do fluxo de dados. A busca por histogramas semelhantes ocorre nos buffers uBOP, nBOP e rBOP. A ordem das pesquisas é a seguinte: 1) NOHAR verifica se existe um histograma semelhante no buffer rBOP, em caso afirmativo, o histograma é classificado e a pesquisa é encerrada; 2) NOHAR verifica se existe um histograma semelhante no buffer nBOP, em caso afirmativo, o histograma é classificado, a pesquisa é encerrada e NOHAR decide se transforma ou não nBOP em rBOP; 3) NOHAR verifica se existe um histograma semelhante no buffer uBOP, em caso afirmativo, NOHAR decide se deve ou não transformar uBOP

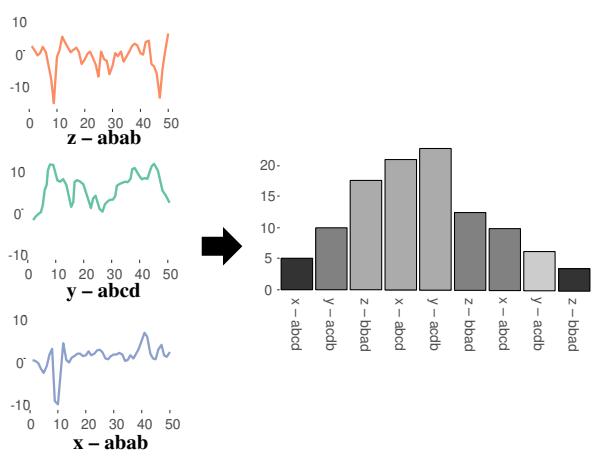

Figure 2: Exemplo de um histograma gerado a partir dos segmentos $\mathrm{x}, \mathrm{y}$ e $\mathrm{z}$.

em nBOP. Se houver mais de um histograma semelhante, NOHAR seleciona o histograma $\mathrm{uBOP}, \mathrm{nBOP}$ ou rBOP mais próximo do histograma não rotulado, ou seja, seleciona o histograma mais próximo.

\section{PROTOCOLO EXPERIMENTAL}

Dividimos os experimentos em dois cenários: (1) NOHAR: Avaliação do algoritmo NOHAR para mostrar uma visão geral detalhada do domínio HAR; (2) NOHAR x AnyNovel [2]: Análise comparativa entre os algoritmos NOHAR e AnyNovel, incluindo desempenho dos modelos de classificação, tempo de processamento e uso do módulo de aprendizagem ativa. O AnyNovel foi escolhido como nossa linha de base principal por três razões: a) É o algoritmo único capaz de detectar novidades; b) Utiliza a aprendizagem ativa para rotular novos conceitos; e c) Foi projetado para lidar com dados de sensores inerciais de smartphones. Os datasets usados nos experimentos são UCI-HAR [5], Shoaib [26] e The Wireless Sensor Data Mining (WISDM) [13].

\section{RESULTADOS - NOHAR X ANYNOVEL}

5.0.1 Análise do modelo de classificação. A Figura 3 mostra uma visão geral da análise comparativa das taxas de precisão dos modelos de classificação obtidos ao longo do tempo por ambos os algoritmos. Como pode ser visto, NOHAR é superior a AnyNovel, em média, $70,12 \%, 72,99 \%$ e $68,30 \%$ para as bases de dados SHOAIB, WISDM e UCI-HAR, respectivamente.

A principal diferença entre NOHAR e AnyNovel é que NOHAR gera modelos de classificação descritivos onde os padrões são capturados com base na estrutura do histograma. Isso significa que os padrões podem ser capturados mesmo quando o conjunto de dados original se cruza no espaço. Por exemplo, sentar e ficar em pé são atividades semelhantes em que os dados dos sensores inerciais possuem interseção no espaço tridimensional. Como o AnyNovel é baseado em clusters dos dados originais, o modelo de classificação se confunde muito com essas atividades. Outra diferença entre os dois algoritmos é que o AnyNovel avalia amostra por amostra, enquanto o NOHAR avalia um conjunto de amostras representadas por histogramas. 

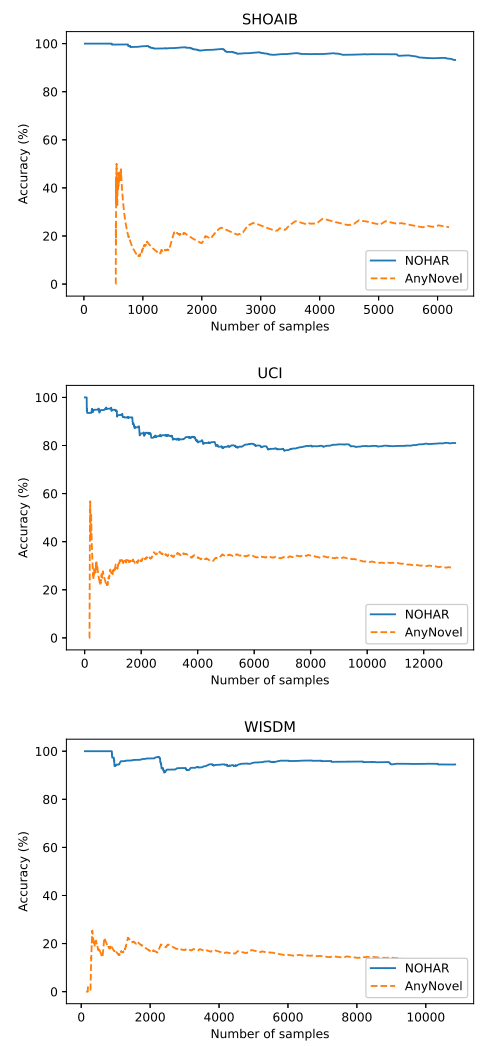

Figure 3: Visão geral das precisões NOHAR e AnyNovel ao longo do tempo para conjuntos de dados SHOAIB, UCI-HAR e WISDM.

5.0.2 Análise do tempo de processamento. A unidade de tempo utilizada é milissegundos (ms) e os resultados são baseados no tempo médio dos algoritmos executados 3 vezes. A Figura 4 mostra que NOHAR levou 4901 ms, 12995 ms e 9912 ms e AnyNovel levou $131782 \mathrm{~ms}, 598092 \mathrm{~ms}$ e $209790 \mathrm{~ms}$ para ser executado nas bases de dados SHOAIB, WISDM e UCI-HAR, respectivamente. Em resumo, o NOHAR é mais rápido que o algoritmo AnyNovel, em média, $96,28 \%, 97,82 \%$ e $95,27 \%$, respectivamente.

Embora as complexidades de ambos os algoritmos sejam lineares no tamanho de entrada, o AnyNovel processa as amostras de um chunk individualmente, diferente do NOHAR que processa a compressão das amostras na forma de histogramas de palavras, bem como o custo de compressão do chunk. Em outras palavras, o AnyNovel processa $n$ instâncias de um chunk. Cada instância é comparada com outras instâncias dentro de $k$ clusters. Para cada cluster ocorre $i$ de iterações. Assim, a complexidade do AnyNovel no tamanho da entrada é $O(n k i)$, desconsiderando a complexidade dos outros componentes que também são lineares [2]. Por outro lado, o NOHAR processa $n$ palavras (frequências) contidas em $k$ histogramas. Para cada histograma ocorre $i$ iterações. Além disso, o custo de discretizar um chunk é $O(n)$, uma vez que o algoritmo usado é SAX [16]. Assim, o custo total da NOHAR é $O(n k i)+O(n)$.
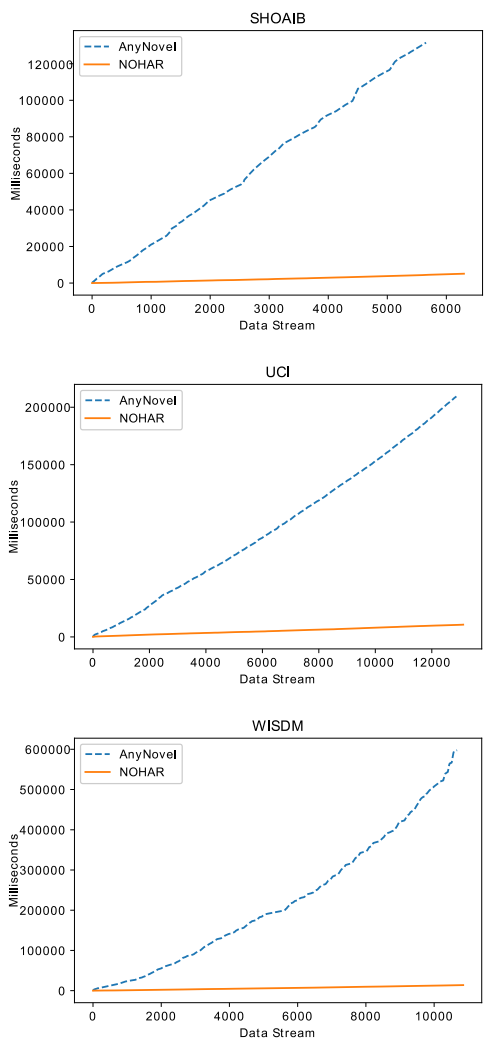

Figure 4: Visão geral do tempo de processamento do NOHAR e AnyNovel nos datasets SHOAIB, WISDM e UCI-HAR.

5.0.3 Análise do consumo de memória. Esta seção apresenta os resultados relativos ao consumo de memória antes e após a discretização dos dados pelo algoritmo NOHAR. Os resultados são descritos na Tabela 1 com unidade de medida Bytes. A estratégia utilizada para extrair esses resultados foi a seguinte: 1) calcular o número de bytes que os dados brutos ocupam na memória; 2) aplicar o processo de discretização e 3) calcular o número de bytes que os dados discretos ocupam na memória.

Table 1: Consumo de memória (bytes) de dados antes e depois do processo de extração de recursos NOHAR.

\begin{tabular}{llll}
\hline Dataset & Dados Brutos & 8 Letras & 4 Letras \\
\hline UCI-HAR & $43,409,405$ & $1,779,486$ & 7,339 \\
WISDM & $22,035,537$ & 866,972 & 5,995 \\
SHOAIB & $16,175,747$ & $1,377,616$ & 7,906 \\
\hline
\end{tabular}

A quantidade de letras das palavras influenciam o consumo de memória física dos dispositivos. Por exemplo, a Tabela 1 mostra os resultados para o SAX parametrizado com 8 e 4 letras. Palavras com 8 letras reduziram o consumo de memória, em média, $94,48 \%$ para todos os datasets, enquanto que palavras com 4 letras reduziram o consumo de memória, em média, 99,97\%. Isso significa uma 
redução de 43.409.405 Bytes dos dados originais para 7.339 Bytes considerando apenas o dataset UCI-HAR. Nestes experimentos, utilizamos palavras com 4 letras. Isso mostra como os algoritmos de representação simbólica são capazes de compactar dados e manter a eficiência dos modelos de classificação e permitir a manipulação de um grande volume de dados.

\section{CONCLUSÕES}

Este artigo propôs uma nova solução adaptativa online composta pelo algoritmo de classificação NOHAR e pelo framework DISTAR. A solução proposta é uma novidade na área de fluxo contínuo de dados porque o NOHAR é o primeiro algoritmo online baseado em algoritmos de representação simbólica. A principal vantagem dessa categoria de algoritmos é a sua capacidade de manipular uma grande quantidade de dados com um custo menor de processamento e consumo de memória. Isso é possível devido à capacidade desses algoritmos em reduzir a dimensionalidade dos dados por meio da compactação de dados pelo processo de discretização.

O caminho que proporcionou a concepção do NOHAR gerou várias publicações de impacto como [29], [28], [27], [9], [18] e [21] Além disso, também tivemos uma publicação de capítulo de livro [4]. Por fim, a versão completa do NOHAR foi publicado aqui [15].

\section{REFERENCES}

[1] Zahraa Said Abdallah, Mohamed Medhat Gaber, Bala Srinivasan, and Shonal Krishnaswamy. 2015. Adaptive mobile activity recognition system with evolving data streams. Neurocomputing 150 (2015), 304-317.

[2] Zahraa Said Abdallah, Mohamed Medhat Gaber, Bala Srinivasan, and Shonali Krishnaswamy. 2016. Anynovel: detection of novel concepts in evolving data streams. Evolving Systems 7, 2 (2016), 73-93.

[3] Ian Anderson, Julie Maitland, Scott Sherwood, Louise Barkhuus, Matthew Chalmers, Malcolm Hall, Barry Brown, and Henk Muller. 2007. Shakra: tracking and sharing daily activity levels with unaugmented mobile phones. Mobile Networks and Applications 12, 2-3 (2007), 185-199.

[4] Thiago Andrade, João Gama, Rita P. Ribeiro, Wesllen Sousa, and André Carvalho. 2019. Anomaly Detection in Sequential Data: Principles and Case Studies American Cancer Society, 1-14. https://doi.org/10.1002/047134608X.W8382 arXiv:https://onlinelibrary.wiley.com/doi/pdf/10.1002/047134608X.W8382

[5] Davide Anguita, Alessandro Ghio, Luca Oneto, Xavier Parra, and Jorge Luis Reyes-Ortiz. 2013. A public domain dataset for human activity recognition using smartphones.. In Esann.

[6] Martin Berchtold, Matthias Budde, Dawud Gordon, Hedda R Schmidtke, and Michael Beigl. 2010. Actiserv: Activity recognition service for mobile phones. In International Symposium on Wearable Computers (ISWC) 2010. IEEE, 1-8.

[7] Albert Bifet and Richard Kirkby. 2011. DATA STREAM MINING A Practical Approach.

[8] Mohd Fikri Azli Bin Abdullah, Ali Fahmi Perwira Negara, Md Shohel Sayeed, Deok-Jai Choi, and Kalaiarasi Sonai Muthu. 2012. Classification algorithms in human activity recognition using smartphones. International fournal of Computer and Information Engineering 6, 77-84 (2012), 106.

[9] Hendrio Bragança, Juan G Colonna, Wesllen Sousa Lima, and Eduardo Souto 2020. A Smartphone Lightweight Method for Human Activity Recognition Based on Information Theory. Sensors 20, 7 (2020), 1856.

[10] Joao Gama. 2010. Knowledge discovery from data streams. Chapman and Hall/CRC.

[11] Chunyu $\mathrm{Hu}$, Yiqiang Chen, Lisha Hu, and Xiaohui Peng. 2018. A novel random forests based class incremental learning method for activity recognition. Pattern Recognition 78 (2018), 277-290.

[12] Ozlem Durmaz Incel, Mustafa Kose, and Cem Ersoy. 2013. A review and taxonomy of activity recognition on mobile phones. BioNanoScience 3, 2 (2013), 145-171.

[13] Jennifer R Kwapisz, Gary M Weiss, and Samuel A Moore. 2011. Activity recognition using cell phone accelerometers. ACM SigKDD Explorations Newsletter 12, 2 (2011), 74-82.

[14] Frédéric Li, Kimiaki Shirahama, Muhammad Nisar, Lukas Köping, and Marcin Grzegorzek. 2018. Comparison of feature learning methods for human activity recognition using wearable sensors. Sensors 18, 2 (2018), 679.

[15] Wesllen Sousa Lima, Hendrio L.S. Bragança, and Eduardo J.P. Souto. 2021. NO HAR - NOvelty discrete data stream for Human Activity Recognition based on smartphones with inertial sensors. Expert Systems with Applications 166 (2021), 114093. https://doi.org/10.1016/j.eswa.2020.114093

[16] Jessica Lin, Eamonn Keogh, Li Wei, and Stefano Lonardi. 2007. Experiencing SAX: a novel symbolic representation of time series. Data Mining and Knowledge Discovery 15, 2 (2007), 107-144.

[17] Emiliano Miluzzo, Cory T Cornelius, Ashwin Ramaswamy, Tanzeem Choudhury, Zhigang Liu, and Andrew T Campbell. 2010. Darwin phones: the evolution of sensing and inference on mobile phones. In Proceedings of the 8th International Conference on Mobile Systems, Applications, and Services. ACM, 5-20.

[18] Kevin G. Montero Quispe, Wesllen Sousa Lima, Daniel Macêdo Batista, and Eduardo Souto. 2018. MBOSS: A Symbolic Representation of Human Activity Recognition Using Mobile Sensors. Sensors 18, 12 (2018). https://doi.org/10.3390/ s18124354

[19] Henry Friday Nweke, Ying Wah Teh, Ghulam Mujtaba, and Mohammed Ali Al-Garadi. 2019. Data fusion and multiple classifier systems for human activity detection and health monitoring: Review and open research directions. Information Fusion 46 (2019), 147-170.

[20] Alan V. Oppenheim. 1975. Digital Signal Processing. Pearson. https://www.xarg. org/ref/a/0132146355/

[21] Kevin G. Montero Quispe, Wesllen Sousa Lima, and Eduardo J. Pereira Souto. 2018. Human Activity Recognition on Smartphones Using Symbolic Data Representation. In Proceedings of the 24th Brazilian Symposium on Multimedia and the Web (WebMedia '18). Association for Computing Machinery, New York, NY, USA, 93-100. https://doi.org/10.1145/3243082.3243116

[22] Patrick Schäfer. 2015. The BOSS is concerned with time series classification in the presence of noise. Data Mining and Knowledge Discovery 29, 6 (2015), 1505-1530.

[23] Patrick Schäfer and Mikael Högqvist. 2012. SFA: a symbolic fourier approximation and index for similarity search in high dimensional datasets. In Proceedings of the 15th International Conference on Extending Database Technology. ACM, 516-527.

[24] Pavel Senin and Sergey Malinchik. 2013. SAX-VSM: Interpretable time series classification using sax and vector space model. Proceedings - IEEE International Conference on Data Mining, ICDM (2013), 1175-1180. https://doi.org/10.1109/ ICDM.2013.52

[25] Jin Shieh and Eamonn Keogh. 2008. i SAX: indexing and mining terabyte sized time series. In Proceedings of the 14th ACM SIGKDD international conference on Knowledge discovery and data mining. ACM, 623-631.

[26] Muhammad Shoaib, Stephan Bosch, Ozlem Incel, Hans Scholten, and Paul Havinga. 2014. Fusion of smartphone motion sensors for physical activity recognition. Sensors 14, 6 (2014), 10146-10176.

[27] Wesllen Sousa, Eduardo Souto, Jonatas Rodrigues, Pedro Sadarc, Roozbeh Jalali, and Khalil El-Khatib. 2017. A comparative analysis of the impact of features on human activity recognition with smartphone sensors. In Proceedings of the $23 \mathrm{rd}$ Brazillian Symposium on Multimedia and the Web. ACM, 397-404.

[28] Wesllen Sousa Lima, Hendrio de Souza Bragança, Kevin Montero Quispe, and Eduardo Pereira Souto. 2018. Human Activity Recognition Based on Symbolic Representation Algorithms for Inertial Sensors. Sensors 18, 11 (2018), 4045.

[29] Wesllen Sousa Lima, Eduardo Souto, Khalil El-Khatib, Roozbeh Jalali, and Joao Gama. 2019. Human activity recognition using inertial sensors in a smartphone: An overview. Sensors 19, 14 (2019), 3213.

[30] Jindong Wang, Yiqiang Chen, Shuji Hao, Xiaohui Peng, and Lisha Hu. 2019. Deep learning for sensor-based activity recognition: A survey. Pattern Recognition Letters 119 (2019), 3-11.

[31] Zhongtang Zhao, Zhenyu Chen, Yiqiang Chen, Shuangquan Wang, and Hongan Wang. 2014. A class incremental extreme learning machine for activity recognition. Cognitive Computation 6, 3 (2014), 423-431. 